\title{
Target Capture via Sliding-Mode Control Incorporating a Reaction Control System
}

\author{
By Hirohisa KoJIMA \\ Tokyo Metropolitan Institute of Technology, Hino, Japan
}

(Received July 22nd, 2002)

\begin{abstract}
The present paper proposes a sliding-mode control method incorporating a strategy to avoid singularities during target capture for a space robot equipped with a manipulator. The position and attitude of the end-effector of the manipulator are controlled by a conventional sliding-mode control employing the transpose of the generalized Jacobian. In the proposed algorithm, the attitude/position of the main body of the space robot is controlled to increase the manipulability when the manipulator approaches a singularity. The direction of the main body translational motion is selected in order to effectively avoid the singularity, and the direction for acceleration of the end-effector is modified taking into account the acceleration of the main body. The gain scheduling technique is also incorporated in order to reduce the control input effort at the beginning of operation as well as to suppress the steady-state error. The effectiveness of the proposed method is verified through numerical simulations for a simple model of a space robot.
\end{abstract}

Key Words: Sliding-Mode Control, Space Robot, Singularity, Debris Elimination

\section{Introduction}

The International Space Station (ISS) has been under construction in orbit since 1997, and Japan, Canada and the EU are currently developing space manipulators for the ISS. Space manipulators will be used to install or replace experimental devices and supply modules, and will be installed on the ISS in a few years. Even with the installation of the space manipulators on the ISS, due to the limited number of astronauts on the ISS, the work time required to complete such tasks is predicted to exceed the time allotted. New technologies are highly desirable for application in space manipulators in order to reduce the number of operations that must be performed by astronauts.

Space robot manipulators are also expected to provide new infrastructures for space development. These types of robot are also referred to as free-flying space robots and have been investigated extensively. ${ }^{1-12)}$ The functions of free-flying space robots include the construction of space structures in orbit and the elimination of space debris from specific orbits, and motion control of the space robot is of critical importance. Control methods of the free-flying space robot are classified into two categories depending on the absence or presence of control of the main body.

For the first category, motion of the manipulator has been studied in order to reduce the possible influence of manipulator motions on the attitude of the main body. Vafa and Dubowsky $^{1,2)}$ have proposed the Virtual Manipulator (VM) method, in which the virtual joint position is calculated geometrically and dynamically so that the motion of the space manipulator can be described using a method equivalent to that of a manipulator on the ground. Dubowsky and Torres $^{3,4)}$ have proposed the Disturbance Map (DM), which

(C) 2003 The Japan Society for Aeronautical and Space Sciences shows the directions of the manipulator motion in joint space that result in the minimum and maximum dynamic disturbances to the main body. In addition, he has proposed a method for planning the effective path of the end-effector using the DM, which results in reduced attitude disturbances to the main body of a free-flying space robot. Nenchev et al. have verified the validity of the non-reaction motion control method, ${ }^{5)}$ in which the manipulator motion does not affect the main body attitude by using the null-space of the reaction motion of the main body attitude from the manipulator motion. This method can be employed when the manipulator has redundancy, that is, the manipulator has more than six degrees of freedom.

With respect to the second category, Kobayashi et al. ${ }^{6}$ have studied an application of sliding-mode control in which the main body reaction due to manipulator motion is eliminated. Although control of attitude/position of the main body is undesirable with respect to energy management, such control could be useful in capturing a target more quickly or safely. Based on this concept, Senda et al. ${ }^{7)}$ have studied various applications of CMG to control the main body attitude of the space robot.

Since the position/attitude of the end-effector must be controlled to capture a target, numerous schemes have been proposed to control the end-effector. These schemes are classified into three primary methods. The first of which uses the inverse matrix of the generalized Jacobian, ${ }^{8)}$ including resolved motion rate control ${ }^{9)}$ and resolved acceleration rate control. ${ }^{10)}$ The second method uses a transpose of the generalized Jacobian, ${ }^{11)}$ and the third method is an application of the sliding-mode control. ${ }^{12)}$

The first method is basically equivalent to the computed torque method and control input cannot be obtained when the generalized Jacobian becomes singular. The position and attitude of the end-effector converge asymptotically to 
the final position and attitude without encountering the singularity problem as the case controlled by the second method. Masutani et al. have proposed a modified method that allows the attitude of the end-effector to be as straight as possible, but the second scheme has a fundamental drawback in that prediction of the time required for target capture is impossible. On the other hand, in the third method, the trajectory of the end-effector is almost a straight line and the convergence time can be predicted from the control gain. The third method has the disadvantage that the end-effector might not reach the target point when a singularity exists on a trajectory of the manipulator or the target point is located out of the workspace of the manipulator. It is difficult to predict whether the end-effector passes through singularities or whether the target point moves out of the workspace of the manipulator before the target is captured, because the target usually moves and rotates relative to the space robot. This disadvantage also occurs in both the first and second control methods.

In order to overcome this problem in the third method, the present paper proposes the modification of a sliding-mode control method incorporating a strategy to avoid manipulator singularities during target capture. The position and attitude of the end-effector are controlled by the conventional sliding-mode control employed with the generalized Jacobian. If the manipulator approaches a singularity, the main body of the space robot changes its position using thrusters so that the manipulability increases. The direction of the thrusters is selected and the guide direction of the end-effector is modified accordingly, taking into consideration the acceleration of the main body in the proposed method in order to increase manipulability effectively. Furthermore, the gain scheduling technique is incorporated in order to reduce the control inputs at the beginning of operation as well as to reduce the steady-state error at the final stage of operation. The effectiveness of the proposed method is verified through numerical simulations.

\section{Sliding-Mode Control for Target Capture}

\subsection{Assumptions}

In order to simplify the target capture problem, the following assumptions are made in the present study:

(1) The parameters of the space robot, such as the length, mass and momentum inertia of each arm, are known exactly.

(2) No dynamic noise affects the space robot.

(3) The relative states of the target with respect to the space robot can be observed correctly.

(4) No time delay exists in the control system.

(5) The manipulator has six degrees of freedom.

\subsection{Discrepancies between target and end-effector}

It is necessary to define the discrepancy between the states of the chaser, such as the end-effector, and those of the target in order to design a tracking control employed with the sliding-mode control. As in numerous previous studies, the present study employs the following variables

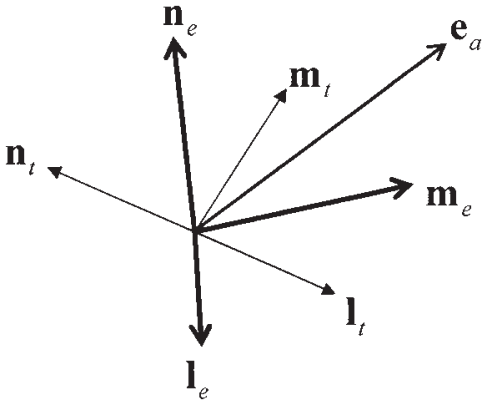

Fig. 1. Attitude errors between target and end-effector.

as the discrepancies:

$$
\boldsymbol{e}=\left[\begin{array}{ll}
\boldsymbol{e}_{\mathrm{p}}^{\mathrm{T}} & \boldsymbol{e}_{\mathrm{a}}^{\mathrm{T}}
\end{array}\right]^{\mathrm{T}},
$$

where $\boldsymbol{e}_{\mathrm{p}}$ and $\boldsymbol{e}_{\mathrm{a}}$ are the position errors and attitude errors, respectively, between the end-effector and the target. The position errors are defined as:

$$
e_{\mathrm{p}}=\boldsymbol{x}_{\mathrm{e}}-\boldsymbol{x}_{\mathrm{t}}
$$

where $\boldsymbol{x}_{\mathrm{e}}$ and $\boldsymbol{x}_{\mathrm{t}}$ are the positions of the end-effector and target, respectively. The attitude errors are defined as:

$$
\boldsymbol{e}_{\mathrm{a}}=\left(\boldsymbol{l}_{\mathrm{e}} \times \boldsymbol{l}_{\mathrm{t}}+\boldsymbol{m}_{\mathrm{e}} \times \boldsymbol{m}_{\mathrm{t}}+\boldsymbol{n}_{\mathrm{e}} \times \boldsymbol{n}_{\mathrm{t}}\right) / 2,
$$

where $\boldsymbol{l}_{\mathrm{e}}, \boldsymbol{m}_{\mathrm{e}}$ and $\boldsymbol{n}_{\mathrm{e}}$ are the axis vectors of the end-effector, and $\boldsymbol{l}_{\mathrm{t}}, \boldsymbol{m}_{\mathrm{t}}$ and $\boldsymbol{n}_{\mathrm{t}}$ are the axis vectors of the target in the coordinate frame of inertia, as shown in Fig. 1. When the attitude of the end-effector coincides with that of the target, the attitude errors, Eq. (3), become equivalent to the zero vector and can be treated as an indicator of attitude errors. The design of a sliding-mode control method in which these discrepancies can be asymptotically eliminated is sufficient if manipulator singularities never occur. This is because the matching condition for the sliding-mode control is automatically satisfied as long as manipulator singularities never occur.

\subsection{Sliding-mode control law using a transpose of the generalized Jacobian ${ }^{12)}$}

If no singularity is included, the following sliding-mode control method can be used to eliminate the preceding discrepancies:

$$
\ddot{\boldsymbol{\theta}}_{\mathrm{m}}=-k \cdot f\left(\boldsymbol{\alpha}, \dot{\boldsymbol{\theta}}_{\mathrm{m}}\right) \boldsymbol{J}_{\mathrm{g}}^{\mathrm{T}} \mathbf{s} \mathbf{a t}(\boldsymbol{\sigma}, \varepsilon),
$$

where $\boldsymbol{\theta}_{\mathrm{m}}$ is the vector of the manipulator joint angles, $f\left(\boldsymbol{\alpha}, \dot{\boldsymbol{\theta}}_{\mathrm{m}}\right)=\alpha_{2}\left\|\dot{\boldsymbol{\theta}}_{\mathrm{m}}\right\|^{2}+\alpha_{1}\left\|\dot{\boldsymbol{\theta}}_{\mathrm{m}}\right\|+\alpha_{0}, \boldsymbol{J}_{\mathrm{g}}$ is the generalized Jacobian, $\sigma$ is the sliding-manifold surface defined as

$$
\sigma=\dot{e}+c e
$$

and $\operatorname{sat}(\sigma, \varepsilon)$ is the saturation function described as

$$
\operatorname{sat}(\sigma, \varepsilon)= \begin{cases}\sigma /\|\sigma\| & \text { if }\|\sigma\| \geq \varepsilon \\ \sigma / \varepsilon & \text { otherwise. }\end{cases}
$$

See the Appendix for the proof of the stability of this control method. The preceding method has an advantage in that the attitude of the end-effector converges to that of the target as the position of the end-effector approaches the target, as shown in Fig. 2. This motion is desirable in order to capture a target smoothly. The method has another advantage in that 


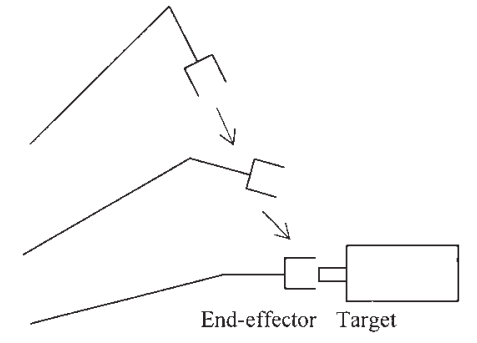

Fig. 2. Manipulator motion by sliding-mode control.

the necessary time can be approximately predicted for discrepancies to converge to zero after getting on the slidingmanifold from the parameters of control.

\section{Strategy to Avoid Manipulator Singularities}

\subsection{Manipulator singularities}

Since the base is fixed for the ground manipulator, the case of the manipulator approaching a singularity is easily estimated. On the other hand, prediction of manipulator singularities is difficult for a free-flying space robot due to the dependence of the singularity on not only the kinematics, but also dynamic parameters, such as the mass and momentum inertia, of the arms. The manipulability of a free-flying space robot, which indicates the ease with which the end-effector can move and rotate, is defined as follows:

$$
M=\sqrt{\operatorname{det}\left(J_{\mathrm{g}} \boldsymbol{J}_{\mathrm{g}}^{\mathrm{T}}\right)} .
$$

Provided that conservation of momentum is satisfied, there exists at least one direction and one axis along which the end-effector cannot move or rotate for the case in which manipulability becomes zero. The generalized Jacobian can be decomposed by Singular Value Decomposition (SVD) as follows:

$$
\boldsymbol{J}_{\mathrm{g}}=\boldsymbol{U} \boldsymbol{W} \boldsymbol{V}^{\mathrm{T}}
$$

where $\boldsymbol{U}$ and $\boldsymbol{V}$ are unitary matrices, $\boldsymbol{W}$ is a diagonal matrix

$$
\boldsymbol{W}=\operatorname{diag}\left(s_{1}, s_{2}, \cdots s_{n}\right), \quad s_{1} \geq s_{2} \cdots \geq s_{n},
$$

and $s_{i}$ is a singular value for the matrix. As a result of this decomposition, a singular direction and a rotation axis can be obtained as a column vector of $\boldsymbol{U}$ corresponding to the singular value, $s_{i}=0$. One of the simplest singularity avoidance methods is to rotate each joint angle. This method is used not only on the ground, but also in space, and is not suitable for automatically controlling space robots to capture targets because the manipulator motion might become discontinuous when the control method changes. Another effective method to avoid singularities is the damped sensitivity least squares (DSLS) method. ${ }^{13)}$ The end-effector controlled by this method, however, cannot trace the design trajectory near singularities precisely, and it is difficult to predict the time required for target capture and prove controller stability when the sliding-mode control is employed in order to control the end-effector of a free-flying space robot using the DSLS matrix rather than the generalized Jacobian. An-

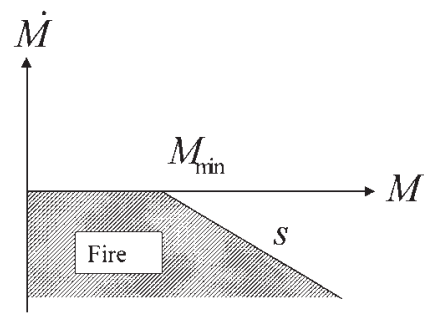

Fig. 3. Phase-plane logic for the RCS of a space robot.

other simple method to avoid singularities is to move the main body, and this method can be applied only to free-flying space robots. In the present study, the last method is utilized in order to avoid manipulator singularities.

\subsection{Design of manipulability phase-plane logic for a space robot $\mathrm{RCS}$}

In the present study, "manipulability phase-plane logic" for the reaction control system (RCS) of a space robot is introduced (Fig. 3). This concept is devised based on the phase-plane logic of the RCS of the Space Shuttle. ${ }^{14)}$ Firing the thrusters is inefficient just after a manipulator singularity has been detected because a short time period exists before the effect of the thruster becomes evident. In addition, firing is not necessary when the manipulator can be easily operated. The manipulability phase plane is therefore divided into two regions. The reaction jets are actuated in the hatched region, and no reaction jet is operated in the blank region in this phase plane. A point, $M_{\min }$, and a slope, $s$, in this map are treated as control parameters.

\subsection{Selection of thruster direction}

In order to effectively avoid manipulator singularities, a simple algorithm to select a reasonable direction for the thruster is introduced. Variation of the manipulability over an infinitesimal period can be approximately described as follows:

$$
\Delta M \approx(\partial M / \partial \boldsymbol{\theta}) \Delta \boldsymbol{\theta}
$$

In order to select the thruster direction simply, the following assumptions are made.

(1) Only one joint rotates in accordance with the movement of the main body;

(2) All joints outside the rotating joint maintain their joint positions in the inertial frame;

(3) All the joints inside the rotating joint lock their joint angles intentionally;

(4) The magnitude of the thruster used to actuate the main body is constant; and

(5) The thruster direction is in the direction of the cross product of the vector of the rotating joint axis and the vector from the main body to the rotating joint.

Under these assumptions, the distance the main body moved during an infinitesimal period, $\Delta d$, can be written as follows:

$$
\Delta d=F \Delta t^{2} / 2 m_{0},
$$

where $F$ is the thruster magnitude and $m_{0}$ is the mass of the main body. The variation of only one rotating joint angle un- 


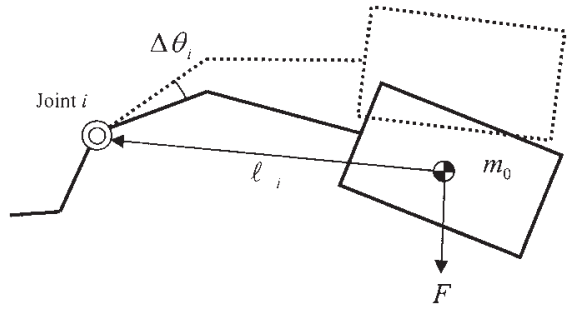

Fig. 4. Selection of thruster-jet direction.

der the preceding assumption (1), in accordance with the movement of the main body during an infinitesimal period, can be obtained as follows:

$$
\Delta \theta_{i}=\Delta d / l_{i}=F \Delta t^{2} / 2 m_{0} l_{i},
$$

where $l_{i}$ is the length from the mass center of the main body to the rotating joint (Fig. 4). Substituting Eq. (12) into Eq. (10), the manipulability variation regarding the rotating joint can be rewritten as follows:

$$
\Delta M_{i} \approx\left(\partial M / \partial \theta_{i}\right) F \Delta t^{2} / 2 m_{0} l_{i}
$$

Consequently, the joint index maximizing $\left(\partial M / \partial \theta_{i}\right) / l_{i}$ should be selected. The following approximate value is used in the present study,

$$
\begin{aligned}
\frac{\partial M_{i}}{\partial \theta_{i}} & \approx \frac{\theta_{i}\left(t_{2}\right)-\theta_{i}\left(t_{1}\right)}{\left\|\boldsymbol{\theta}\left(t_{2}\right)-\boldsymbol{\theta}\left(t_{1}\right)\right\|_{2}}\left\{M\left(t_{2}\right)-M\left(t_{1}\right)\right\}, \\
\Delta t & =t_{2}-t_{1},
\end{aligned}
$$

because the exact derivative of the manipulability with respect to each joint angle is difficult to obtain. Note that the preceding situations are simply assumptions for determining the effective thruster direction in a simple manner. The deviation of manipulability with respect to all joint angles for a short duration is calculated and the effectiveness of the proposed method will be verified in numerical simulations.

In this study, the thruster is operated only for the case in which manipulability is near singularity and approaches singularity more closely. When the attitude of the main body is changed tentatively due to the thruster firing and this change leads to difficulty in approaching the target, this state will be sensed as the singularity for the manipulator. In the proposed algorithm, the main body is moved again in such a case by firing the thruster to approach the target easily. Accordingly, even if the thrust vector does not completely pass the center of mass of the main body, the motion of the main body induced by the thruster firing results in the desirable direction in which the target will be captured easily when the thrust vector passes near the center of the mass of the main body. Thus, the tentative attitude changes of the main body do not become a severe problem. The operation range and shape restriction of the manipulator need to be considered generally, but are not investigated in this study for the sake of simplicity.

\subsection{Modification of acceleration direction of the end- effector}

Actuation of the main body motion disturbs the accelera-

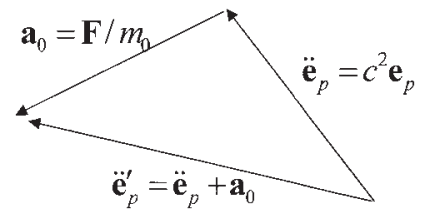

Fig. 5. Modification of relative position.

tion direction of the end-effector, and modification of the acceleration direction of the end-effector is necessary when the thruster accelerates the main body. The only situation that needs be considered is that the end-effector state is already positioned on the sliding-manifold surface. This is because the state of the end-effector can be forced to converge on the manifold surface within a finite time by the slidingmode control even if the state is far from the manifold surface. When thrusters are not used to move the main body and the end-effector state is already on the sliding manifold, the relative acceleration direction of the end-effector to the target can be written as follows:

$$
\ddot{\boldsymbol{e}}_{\mathrm{p}}=c^{2} \boldsymbol{e}_{\mathrm{p}}
$$

In order to counteract the effect of the thrusters used to accelerate the main body on the acceleration direction of the end-effector, the position of the end-effector relative to the target, $\boldsymbol{e}_{\mathrm{p}}$ in Eq. (1), should be replaced with $\boldsymbol{e}_{\mathrm{p}}^{\prime}$ as follows:

$$
\boldsymbol{e}_{\mathrm{p}}^{\prime}=\frac{\ddot{\boldsymbol{e}}_{\mathrm{p}}^{\prime}}{c^{2}}=\frac{c^{2} \boldsymbol{e}_{\mathrm{p}}+\boldsymbol{a}_{0}}{c^{2}}=\boldsymbol{e}_{\mathrm{p}}+\frac{\boldsymbol{F}}{m_{0} c^{2}},
$$

where $\boldsymbol{a}_{0}$ is the acceleration direction of the main body due to the thrusters (Fig. 5).

\subsection{Gain scheduling}

Sliding-mode control has a tendency whereby the control input at the beginning of the operation increases, and this tendency is often undesirable for actuators with limited magnitudes. The control law, Eq. (4), has another drawback in that the steady-state error, of at most $\varepsilon / c$, might remain (see Eq. (A23)). The possible steady-state error will become smaller, but the control input at the beginning of operation becomes larger if parameter $c$ is chosen to be larger. As a compromise to address this problem, the gain scheduling technique should be employed (i.e., parameter $c$ should be set small at the beginning of the operation, and should become larger as the magnitude of the sliding vector becomes smaller). The value of parameter $c$ should also change smoothly in accordance with the absolute magnitude of the error states in order to avoid discontinuity of the control input. In the present study, the following nonlinear slidingmanifold function is employed to schedule slope parameter $c$ in accordance with the magnitude of the error state:

$$
\boldsymbol{\sigma}=\dot{\boldsymbol{e}}+C(\boldsymbol{e}) \boldsymbol{e},
$$

where

$$
C(\boldsymbol{e}) \equiv 2.0 c\left(1.4-\frac{1}{1+\exp (-a(\|\boldsymbol{e}\|-b))}\right) .
$$

Figure 6 illustrates the examples of sliding-manifold surfaces $\left(\sigma_{i}=0\right)$ on the phase plane of the error state derived 


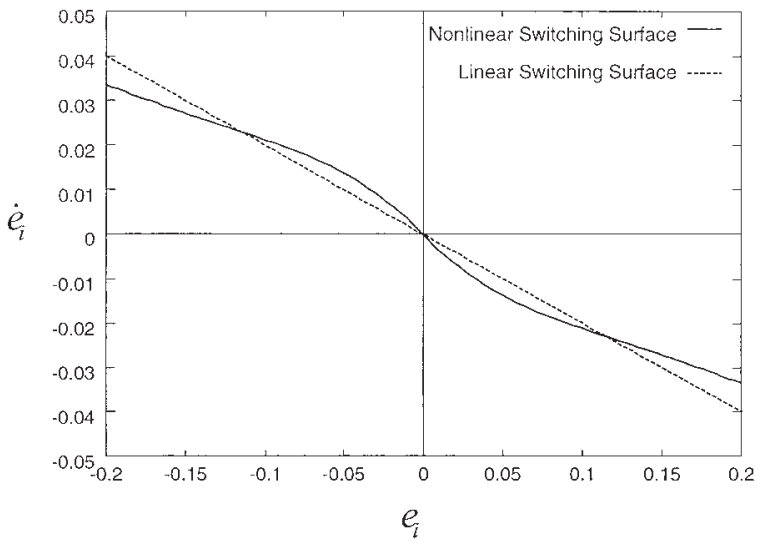

Fig. 6. Sliding-manifold surface.

from Eqs. (5) and (17). As shown in Fig. 6, the slope of the nonlinear switching surface becomes greater than that of the linear switching surface as the error state becomes smaller.

\section{Numerical Simulations}

\subsection{Schematic model and parameters used for simula- tion}

Figure 7 shows a model of the space robot discussed in the present study. The mass and inertia tensor of each arm are listed in Table 1. These parameters are the same as those of Ref. 11). The second and third arms are longer than the other arms and are primarily used to adjust the position of

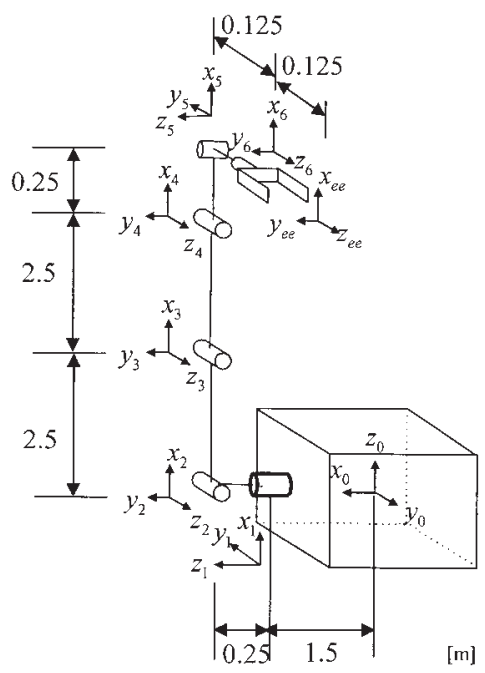

Fig. 7. The free-flying space robot.

Table 1. Parameters of the space robot manipulator.

\begin{tabular}{ccll}
\hline No. & Mass $[\mathrm{kg}]$ & \multicolumn{2}{c}{$I x, I y, I z\left[\mathrm{kgm}^{2}\right]$} \\
\hline 0 & 2000 & $1400, \quad 1400,2040$ \\
1 & 20 & $0.15, \quad 0.10, \quad 0.15$ \\
2 & 50 & $0.25, \quad 26.0,26.0$ \\
3 & 50 & $0.25, \quad 26.0, \quad 26.0$ \\
4 & 10 & $0.05, \quad 0.075,0.075$ \\
5 & 5 & $0.025,0.025,0.019$ \\
6 & 5 & $0.025,0.025,0.025$ \\
\hline
\end{tabular}

Table 2. Parameters of simulation.

\begin{tabular}{cc}
\hline Symbol & Value [Unit] \\
\hline$\varepsilon$ & $0.2[1 / \mathrm{s}]$ \\
$k$ & 0.002 \\
$\alpha$ & $2.0[1 / \mathrm{s}]$ \\
$F$ & {$[0.5,0.2,0.003]$} \\
$M_{\min }$ & $200[\mathrm{~N}]$ \\
$s$ & $0.88\left[\mathrm{~m}^{3}\right]$ \\
$\Delta t$ & $-5.0[1 / \mathrm{s}]$ \\
$a$ & $0.005[\mathrm{~s}]$ \\
$b$ & 20 \\
\end{tabular}

the end-effector. The three arms near the end-effector are used primarily to adjust the attitude of the end-effector with respect to the target. The parameters of simulation, including control gains, are listed in Table 2. The initial angle and angular velocity of each joint are given as $(0,0.2$, $\pi / 2,0, \pi / 2,0)[\mathrm{rad}]$ and $(0,0,0,0,0,0)[\mathrm{rad} / \mathrm{s}]$, respectively. The initial position, velocity, angular velocity and Euler parameters of the main body are set as $(0,0,0)[\mathrm{m}],(0,0,0)$ $[\mathrm{m} / \mathrm{s}],(0,0,0)[\mathrm{rad} / \mathrm{s}]$ and $(0,0,0,1)$, respectively. The target is assumed to be in equilibrium state, and its position and Euler parameters are set to $(2.9,0,-3.7)[\mathrm{m}]$ and $(0$, $-\sqrt{2} / 2,0, \sqrt{2} / 2)$, respectively. The target satellite is assumed to be cylindrical, with a radius of $0.5[\mathrm{~m}]$ and a height of $1.0[\mathrm{~m}]$, and is assumed to be captured at the center of its bottom face. The straight-line segment connecting the initial position of the end-effector and the target point has no intersection with the target shape at this initial condition.

\subsection{Simulation results}

In order to demonstrate the effectiveness of the proposed method, four types of control methods are analyzed numerically in the present study. One method studies the case in which the manipulator is controlled by conventional sliding-mode control. ${ }^{12)}$ The second includes the manipulability phase-plane logic and selection of thruster direction for singularity avoidance, but excludes modification of the end-effector acceleration and gain scheduling technique. The third includes the proposed strategy for singular avoidance without the gain scheduling technique. The fourth simulation includes all of the proposed strategies. Hereafter, the first, second, third and fourth simulations are referred to as Case-1, Case-2, Case-3 and Case-4, respectively. The fourth-order Runge-Kutta method is used to integrate the equations of motion associated with each control scheme.

Figures 8, 9, 10 and 11 show the time responses of the error states for Case-1, Case-2, Case-3 and Case-4, respectively. The errors are seen to remain in Case-1, but are almost eliminated in Case-2, Case-3 and Case-4. The time responses of manipulability for Case-1, Case-2, Case- 3 and Case- 4 are shown in Figs. 12, 13, 14 and 15, respectively. Numerous manipulator singularities occur for Case-1, but only twice for Case- 2 and only once for Case- 3 and Case-4. Some of error states increase shortly after the start of manipulator operation. This is because the stability of the sliding-mode 


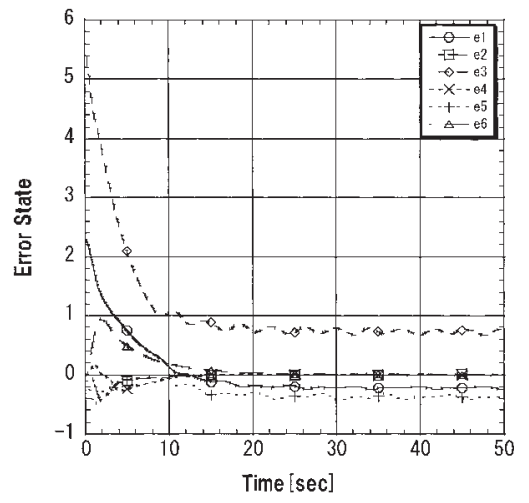

Fig. 8. Time response of error states for Case-1.

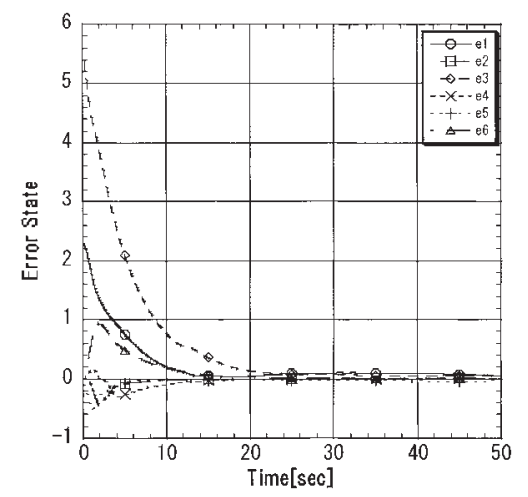

Fig. 9. Time response of error states for Case-2.

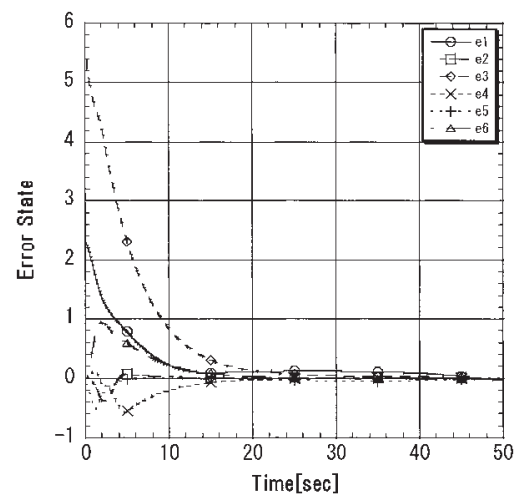

Fig. 10. Time response of error states for Case-3.

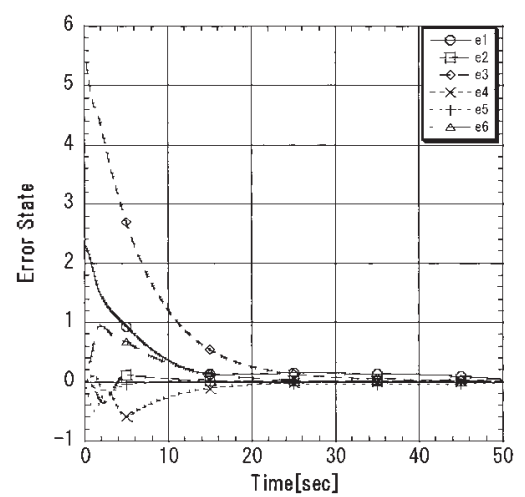

Fig. 11. Time response of error states for Case-4.

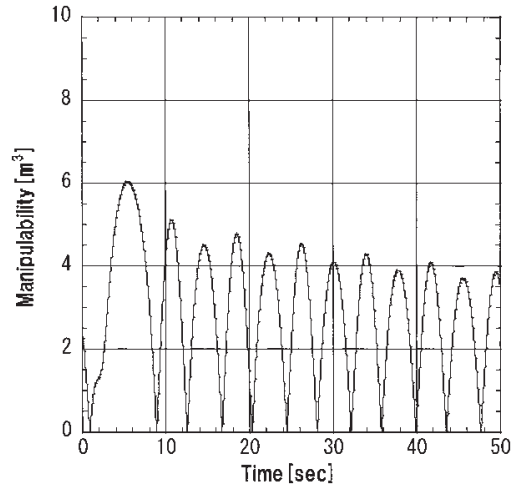

Fig. 12. Time response of manipulability for Case-1.

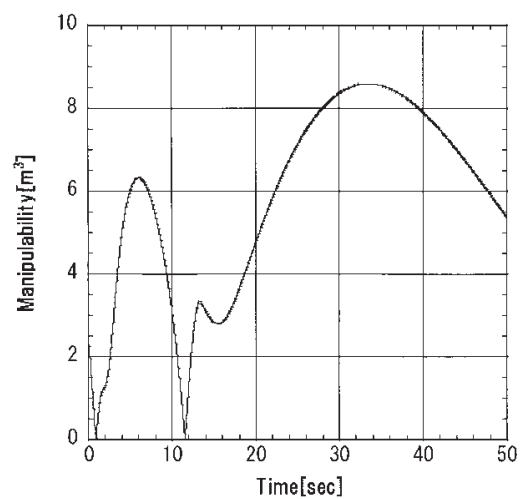

Fig. 13. Time response of manipulability for Case-2.

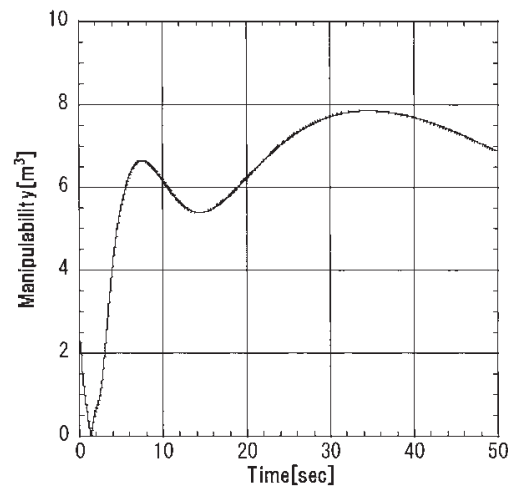

Fig. 14. Time response of manipulability for Case-3.

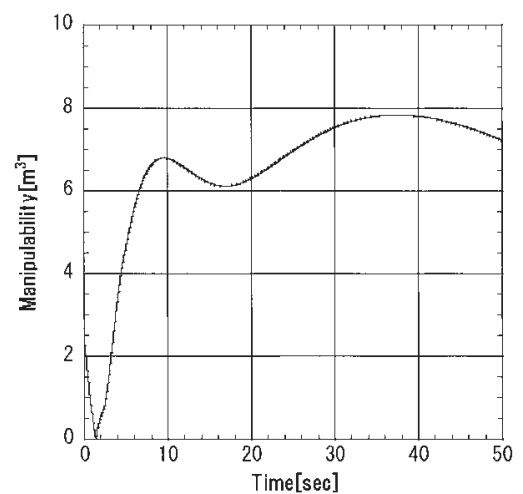

Fig. 15. Time response of manipulability for Case-4. 

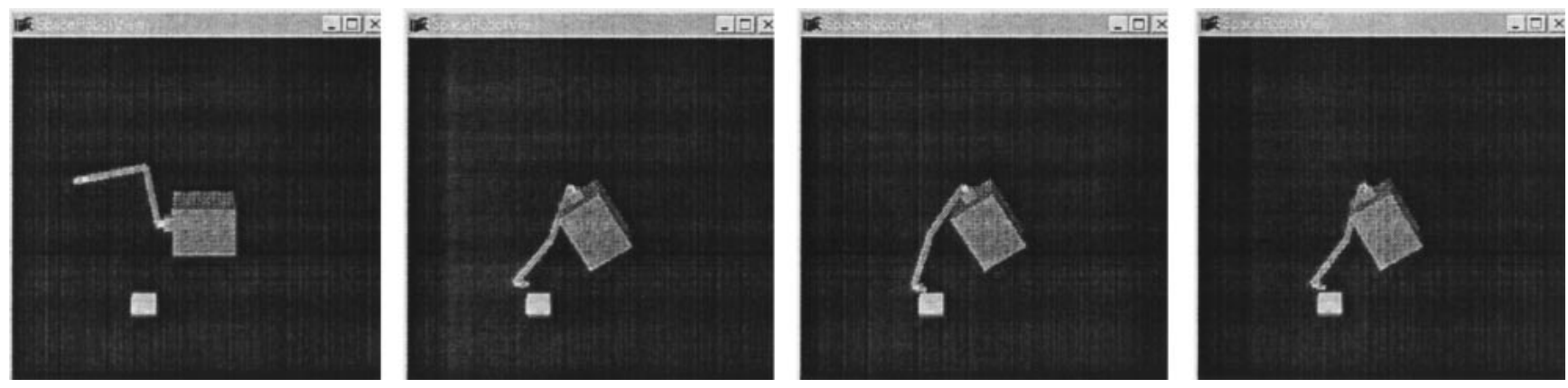

Fig. 16. Animation of the space robot motion for Case-1.
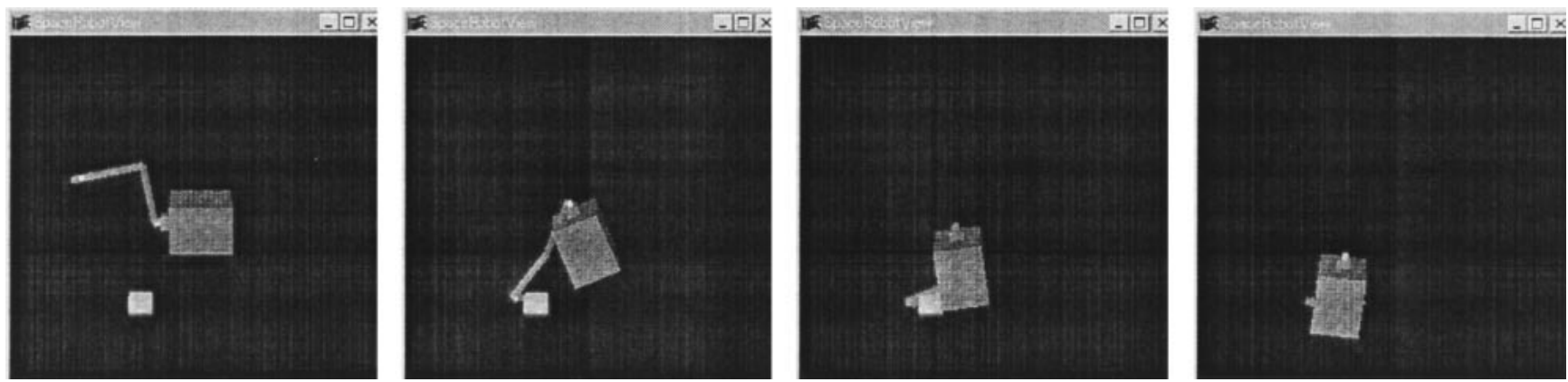

Fig. 17. Animation of the space robot motion for Case-2.
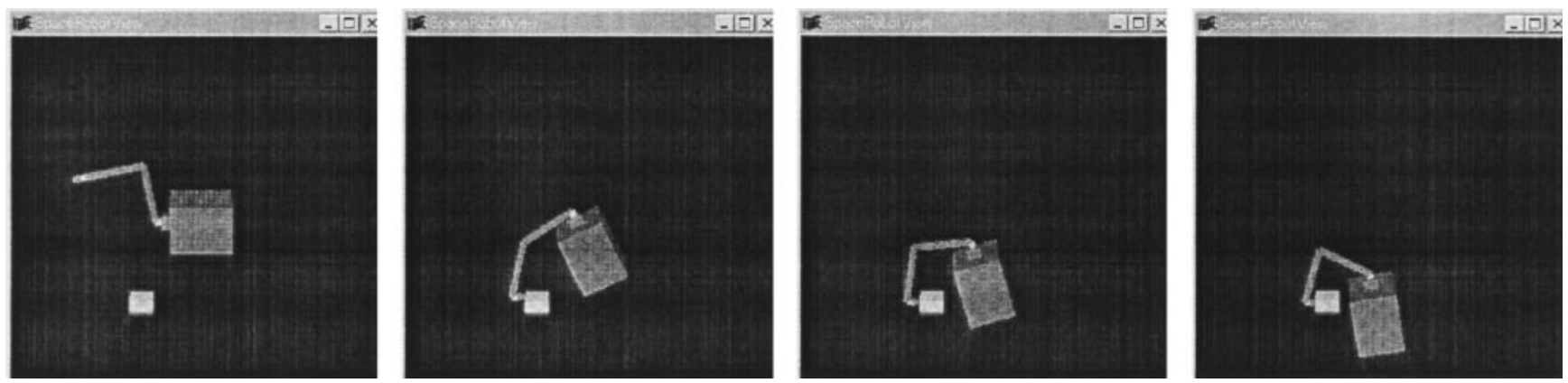

Fig. 18. Animation of the space robot motion for Case-3.
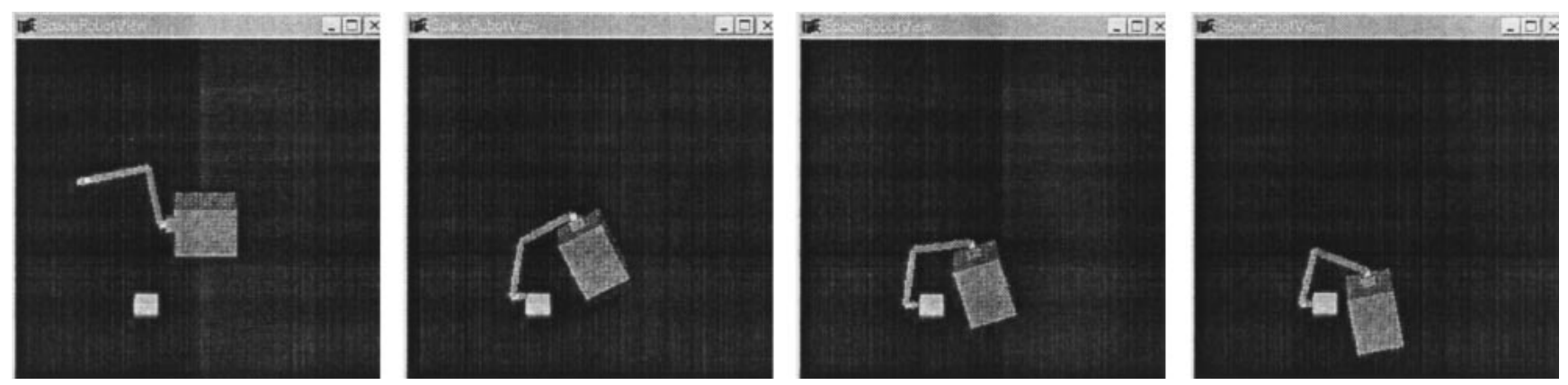

Fig. 19. Animation of the space robot motion for Case-4.

controller is guaranteed only when the manipulability is greater than some value determined from the value chosen for parameter $k$. Figures 16, 17, 18 and 19 are animations of the manipulator motion for Case-1, Case-2, Case-3 and Case-4, respectively. As shown in Fig. 16, a limit cycle occurs in the vicinity of singularity for Case-1. The target cannot be reached by the end-effector using traditional slidingmode control that does not include a singularity avoidance strategy. In other words, the target is located out of the workspace of the end-effector for the initial state as described above. The end-effector for Case-2 is seen to reach the target, but the thruster was used to actuate the main body so much that the main body collided with the target. This is because the effect of the thruster jet on the motion of the end-effector is not taken into account in the control law for Case-2. On the contrary, the main body does not collide 


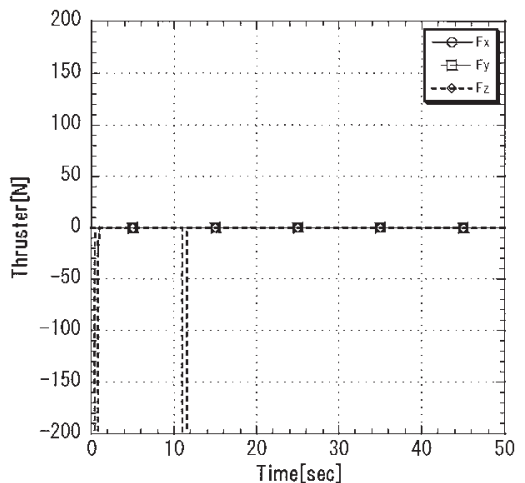

Fig. 20. Time response of thruster jets for Case-2.

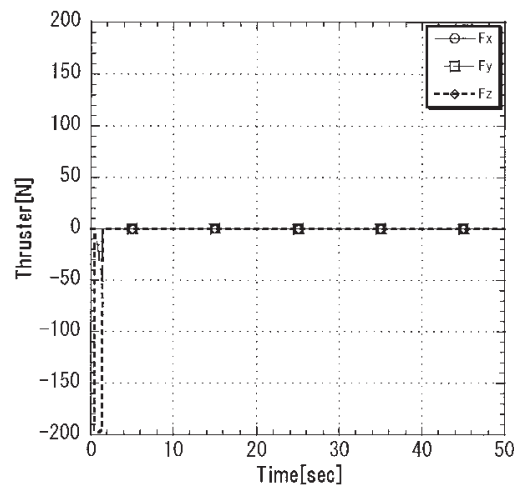

Fig. 21. Time response of thruster jets for Case-3.

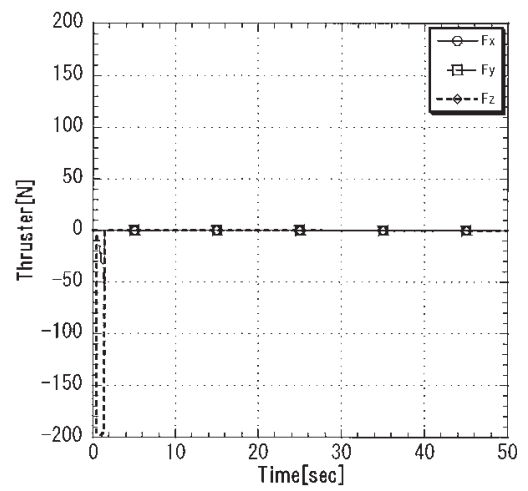

Fig. 22. Time response of thruster jets for Case-4.

with the target for Case-3 and Case-4, as shown in Figs. 18 and 19. Time responses of control input (thrusters) for Case2, Case- 3 and Case-4 are shown in Figs. 20, 21 and 22, respectively. It can be seen that thrusters are operated when manipulability is approaching zero, and that thruster operation stops after manipulability increases. It can also be seen that the number of thruster jet operations for Case- 3 and Case- 4 is less than that for Case-2. Unnecessary thruster jet operation is avoided for Case- 3 and Case- 4 by tuning the end-effector motion in accordance with the main body motion actuated by the thruster jet. Regarding the gain scheduling technique, a comparison between Fig. 10 and Fig. 11 indicates that error states for Case- 4 decrease slightly more slowly than those for Case-3 during the first half of the operation, and steady errors for Case- 4 are finally reduced to be as small as those for Case-3. In addition, the de- crease in manipulability after reaching the first peak for Case-4 is not greater than that for Case-3. This is because the manipulability for Case-4 approaches the singularity more slowly than that for Case-3 after it reaches the first peak, and as a result, the thruster jet for Case-4 was more effective than that of Case-3. The gain scheduling technique employed in the present study reduces the number of manipulator singularities.

It is necessary to avoid interference between the effects of the control system for main body attitude and the effects of the end-effector control system after target capture, because the total system configuration changes when the end-effector captures the target, and such a change might cause an unexpected problem. Such issues will be taken into consideration in order to confirm safer captures in the future. It is also necessary to study methods by which to prevent the thruster jet of the space robot from contaminating the target when controlling the position of the space robot.

\section{Conclusions}

The present paper has introduced a sliding-mode control method which contains a strategy to avoid manipulator singularities. The position and attitude of the end-effector are controlled using the traditional sliding-mode control based on the generalized Jacobian, and the main body of the space robot is repositioned using thrusters so that manipulability increases when the manipulator approaches a singularity. In order to increase manipulability effectively, the direction of the thrusters is selected and the guide direction of the endeffector is modified accordingly, taking into account acceleration of the main body. A gain scheduling technique has been employed in order to reduce the steady errors between the end-effector and the target, and to reduce the magnitude of the control input at the beginning of the capturing operation. The effectiveness of the proposed method has been demonstrated through numerical simulations and by comparison of the results employed with the conventional sliding-mode control.

Future research will examine the range of joint angle and the berthing problem after the target is captured.

\section{Acknowledgments}

The present study was supported in part by a Grand-in-Aid for Scientific Research (No. 14550855) from the Ministry of Education, Culture, Sports, Science, and Technology of Japan.

\section{References}

1) Vafa, Z. and Dubowsky, S.: On the Dynamics of Manipulators in Space Using the Virtual Manipulator Approach, Proceedings of IEEE International Conference on Robotics and Automation, 1987, pp. 579585.

2) Vafa, Z. and Dubowsky, S.: On the Dynamics of Space Manipulator Using the Virtual Manipulator with Application to Path Planning, $J$. Astronaut. Sci., 38 (1990), pp. 441-472.

3) Dubowsky, S. and Torres, M. A.: Path Planning for Space Manipulator to Minimize Spacecraft Attitude Disturbance, Proceedings of the 
IEEE International Conference on Robotics and Automation, 1991, pp. 2522-2528.

4) Torres, M. and Dubowsky, S.: Minimizing Spacecraft Attitude Disturbance in Space Manipulator Systems, J. Guid. Control Dynam., 15 (1992), pp. 1010-1017.

5) Nenchev, D. N., Yoshida, K., Vichitkulsawat, P., Konno, A. and Uchiyama, M.: Experiments on Reaction Null-Space Based Decoupled Control of a Flexible Structure Mounted Manipulator System, Proceedings of the 1997 IEEE International Conference on Robotics and Automation, 1997, pp. 2528-2534.

6) Kobayashi, N., Nonami, K., Yasuzumi, I. and Nakayama, T.: Attitude Control Free-Flying Space Robot with Discrete Time Sliding Mode Control Algorithm, Trans. Soc. Instrum. Control Eng., 32 (1996), pp. 1206-1211 (in Japanese).

7) Senda, K., Murotsu, Y., Nagaoka, H. and Mitsuya, A.: Attitude Control for Free-Flying Space Robot with CMG, AIAA Paper-95-3336, Proceedings of AIAA Guidance, Navigation, and Control Conference, 1995, pp. 1492-1502.

8) Umetani, Y. and Yoshida, K.: Continuous Path Control of Space Manipulators Mounted on OMV, Acta Astronautica, 15 (1987), pp. 981986.

9) Umetani, Y. and Yoshida, K.: Resolved Motion Rate Control of Space Manipulators with Generalized Jacobian Matrix, IEEE Trans. Rob. Autom., 5 (1989), pp. 303-314.

10) Longman, R. W., Lindberg, R. E. and Zedd, M. F.: Satellite-Mounted Robot Manipulators-New Kinematics and Reaction Moment Compensation, Int. J. Rob. Res., 6 (1987), pp. 87-103.

11) Masutani, Y., Miyazaki, F. and Arimoto, S.: Sensory Feedback Control for Space Manipulators, Proceedings of IEEE International Conference on Robotics and Automation, 1989, pp. 1346-1351.

12) Harima, H., Kawaguchi, J., Nakatani, I. and Ninomiya, K.: Control of Space Manipulator Using Sliding Mode Control, J. Robot, 9 (1991), pp. 572-579 (in Japanese).

13) Nakamura, Y. and Hanafusa, H.: Singularity Low-Sensitive Motion of Articulated Robot Arms, SICE, 20 (1984), pp. 453-459 (in Japanese).

14) Wie, B.: Space Vehicle Dynamics and Control, AIAA, Reston, 1998, pp. 445-451.

\section{Appendix: Proof of Stability of Sliding-Mode Con- trol $^{12)}$}

\section{A.1. Evaluation of absolute magnitude}

The law of conservative momentum and angular momentum is as follows:

$$
H_{x_{0}} \dot{x}_{0}+H_{\theta} \dot{\theta}_{\mathrm{m}}=H_{q} q,
$$

where $\dot{x}_{0}$ represents the velocity and angular velocity of the main body, $\dot{\theta}_{\mathrm{m}}$ is the rotational velocities of the manipulator joints, $H_{x_{0}}$ are $H_{\theta}$ the momentum and angular momentum Jacobian matrices of the system, respectively, and $q$ is a vector representing the momentum and angular momentum. The derivative of the preceding equation with respect to time is obtained as follows:

$$
H_{x_{0}} \ddot{x}_{0}+H_{\theta} \ddot{\theta}_{\mathrm{m}}+\dot{H}_{x_{0}} \dot{x}_{0}+\dot{H}_{\theta} \dot{\theta}_{\mathrm{m}}=\dot{H}_{q} q+H_{q} \dot{q} .
$$

The acceleration/angular acceleration of the main body is written using the preceding equation as follows:

$$
\ddot{x}_{0}=-H_{x_{0}}^{-1}\left(H_{\theta} \ddot{\theta}_{\mathrm{m}}+\dot{H}_{x_{0}} \dot{x}_{0}+\dot{H}_{\theta} \dot{\theta}_{\mathrm{m}}-\dot{H}_{q} q-H_{q} \dot{q}\right) .
$$

The acceleration of the end-effector is represented as

$$
\ddot{x}_{\mathrm{ee}}=\dot{J} \dot{\theta}+J \ddot{\theta},
$$

where $J$ is the kinematic Jacobian and $\dot{\theta}$ is the set of velocity/angular velocity of the main body and rotational velocity of the manipulator joints. The second term in the right-hand side of Eq. (A4) can be rewritten as follows:

$$
\begin{aligned}
J \ddot{\theta} & =J_{\mathrm{s}} \ddot{x}_{0}+J_{\mathrm{m}} \ddot{\theta}_{\mathrm{m}} \\
& =J_{\mathrm{g}} \ddot{\theta}_{\mathrm{m}}-J_{\mathrm{s}} H_{x_{0}}^{-1}\left(\dot{H}_{\theta} \dot{\theta}_{\mathrm{m}}+\dot{H}_{x_{0}} \dot{x}_{x_{0}}-\dot{H}_{q} q\right),
\end{aligned}
$$

where $J_{\mathrm{g}}$ is the generalized Jacobian and $J_{\mathrm{s}}$ and $J_{\mathrm{m}}$ are Jacobian matrices with respect to the velocity/angular velocity of the main body and joint velocities of the manipulator, respectively. The absolute magnitude of the velocity/angular velocity of the main body can be evaluated as :

$$
\begin{aligned}
\left\|\dot{x}_{0}\right\| & =\left\|-H_{x_{0}}^{-1}\left(H_{x_{0}} \dot{x}_{0}+H_{\theta} \dot{\theta}_{\mathrm{m}}-H_{q} q\right)\right\| \\
& \leq\left\|H_{x_{0}}^{-1}\right\|\left\|H_{\theta}\right\|\left\|\dot{\theta}_{\mathrm{m}}\right\|+\left\|H_{x_{0}}^{-1} H_{q} q\right\| .
\end{aligned}
$$

Thus, the following evaluation is obtained:

$$
\begin{gathered}
\left\|J_{\mathrm{s}} H_{x_{0}}^{-1}\left(\dot{H}_{\theta} \dot{\theta}_{\mathrm{m}}+\dot{H}_{x_{0}} \dot{x}_{x_{0}}-\dot{H}_{q} q\right)\right\| \\
\leq \beta_{21}\left\|\dot{\theta}_{\mathrm{m}}\right\|^{2}+\beta_{11}\left\|\dot{\theta}_{\mathrm{m}}\right\|+\beta_{01} .
\end{gathered}
$$

The first term of the right-hand side of Eq. (A4) can be evaluated as:

$$
\begin{aligned}
\|\dot{\boldsymbol{J}} \dot{\theta}\| & \leq\|\dot{\boldsymbol{J}}\|\|\dot{\theta}\| \leq\left\|\sum \dot{\theta}_{i} \frac{\partial J}{\partial \theta_{i}}\right\|\|\dot{\theta}\| \leq\left\|\sum \frac{\partial J}{\partial \theta_{i}}\right\|\|\dot{\theta}\|^{2} \\
& \leq \beta_{22}\left\|\dot{\theta}_{\mathrm{m}}\right\|^{2}+\beta_{12}\left\|\dot{\theta}_{\mathrm{m}}\right\|+\beta_{02} .
\end{aligned}
$$

The velocity of the end-effector is represented as:

$$
\dot{x}_{\mathrm{ee}}=J \dot{\theta}=J_{x_{0}} \dot{x}_{0}+J_{\mathrm{m}} \dot{\theta}_{\mathrm{m}}=J_{\mathrm{g}} \dot{\theta}_{\mathrm{m}}+J_{x_{0}} H_{x_{0}}^{-1} H_{q} q,
$$

Thus, the absolute magnitude of the velocity of the end-effector can be evaluated as:

$$
c\left\|\dot{x}_{\mathrm{ee}}\right\| \leq c\left\|J_{\mathrm{g}} \dot{\theta}_{\mathrm{m}}+J_{x_{0}} H_{x_{0}}^{-1} H_{q} q\right\| \leq \beta_{13}\left\|\dot{\theta}_{\mathrm{m}}\right\|+\beta_{03} .
$$

Furthermore, if no external force or torque affects the target, the absolute magnitude of the acceleration and velocity of the target can be evaluated as:

$$
\left\|-\ddot{x}_{\mathrm{t}}-c \dot{x}_{t}\right\| \leq \beta_{04} .
$$

The derivative of the sliding vector with respect to the time, $\dot{\boldsymbol{\sigma}}$, is obtained as follows:

$$
\dot{\boldsymbol{\sigma}}=J \ddot{\theta}+\dot{J} \dot{\theta}-\ddot{x}_{\mathrm{t}}+c\left(\dot{x}_{\mathrm{ee}}-\dot{x}_{\mathrm{t}}\right) .
$$

Substituting the preceding evaluations, Eqs. (A7), (A8), (A10) and (A11) into Eq. (A12), the magnitude of the derivative of the sliding vector can be evaluated as follows:

$$
\left[J_{\mathrm{g}} \ddot{\theta}_{\mathrm{m}}\right]_{i}-f\left(\boldsymbol{\beta}, \dot{\theta}_{\mathrm{m}}\right) \leq \dot{\sigma}_{i} \leq\left[J_{\mathrm{g}} \ddot{\theta}_{\mathrm{m}}\right]_{i}+f\left(\boldsymbol{\beta}, \dot{\theta}_{\mathrm{m}}\right),
$$

where

$$
\begin{aligned}
f\left(\boldsymbol{\beta}, \dot{\theta}_{\mathrm{m}}\right) & =\beta_{2}\left\|\dot{\theta}_{\mathrm{m}}\right\|^{2}+\beta_{1}\left\|\dot{\theta}_{\mathrm{m}}\right\|+\beta_{0} \\
\beta_{2} & =\beta_{21}+\beta_{22} \\
\beta_{1} & =\beta_{11}+\beta_{12}+\beta_{13} \\
\beta_{0} & =\beta_{01}+\beta_{02}+\beta_{03}+\beta_{04} .
\end{aligned}
$$

The parameters $k$ and $\alpha$ in the control law, Eq. (4), are chosen such that the following conditions are satisfied: 


$$
\begin{gathered}
k>1 / \sigma_{\mathrm{m}}\left(J_{\mathrm{g}} J_{\mathrm{g}}^{\mathrm{T}}\right) \\
\alpha: \alpha_{i}>\beta_{i} \quad(i=2,1,0),
\end{gathered}
$$

where $\sigma_{\mathrm{m}}(x)$ denotes the minimum singular value of $x$.

Substituting Eq. (4) into Eq. (12) yields

$$
\begin{aligned}
& {\left[-k f\left(\boldsymbol{\alpha}, \dot{\theta}_{\mathrm{m}}\right) J_{\mathrm{g}} J_{\mathrm{g}}^{\mathrm{T}} \operatorname{sat}(\boldsymbol{\sigma}, \boldsymbol{\varepsilon})\right]_{i}-f\left(\boldsymbol{\beta}, \dot{\theta}_{\mathrm{m}}\right)} \\
& \quad \leq \dot{\boldsymbol{\sigma}}_{i} \leq\left[-k f\left(\boldsymbol{\alpha}, \dot{\theta}_{\mathrm{m}}\right) J_{\mathrm{g}} J_{\mathrm{g}}^{\mathrm{T}} \operatorname{sat}(\boldsymbol{\sigma}, \boldsymbol{\varepsilon})\right]_{i}+f\left(\boldsymbol{\beta}, \dot{\theta}_{\mathrm{m}}\right) .
\end{aligned}
$$

It will now be proven that the reaching mode and sliding mode can be achieved by the sliding-mode controller, Eq. (4).

A candidate Lyapunov function is choosen as follows:

$$
V=\boldsymbol{\sigma}^{\mathrm{T}} \boldsymbol{\sigma} / 2=\frac{1}{2} \sum_{i} \sigma_{i} \sigma_{i}=\sum_{i} V_{i} .
$$

The derivative of the candidate Lyapunov function is obtained as:

$$
\dot{V}=\sigma^{\mathrm{T}} \dot{\boldsymbol{\sigma}}=\sum_{i} \sigma_{i} \dot{\sigma}_{i}=\sum_{i} \dot{V}_{i}
$$

\section{A.2. Reaching mode}

The case $\|\sigma\| \geq \varepsilon$ is assumed in this subsection in order to prove the existence of the reaching mode. If $\sigma_{i}>0$, the derivative of the $i$ th part of the candidate Lyapunov function can be evaluated as follows:

$$
\begin{aligned}
\dot{V}_{i} & =\sigma_{i} \dot{\sigma}_{i} \leq \sigma_{i}\left[-k f\left(\boldsymbol{\alpha}, \dot{\theta}_{\mathrm{m}}\right) J_{\mathrm{g}} J_{\mathrm{g}}^{\mathrm{T}} \operatorname{sat}(\boldsymbol{\sigma}, \varepsilon)\right]_{i}+f\left(\boldsymbol{\beta}, \dot{\theta}_{\mathrm{m}}\right) \\
& \leq-f\left(\boldsymbol{\alpha}, \dot{\theta}_{\mathrm{m}}\right) \sigma_{i} \sigma_{\mathrm{m}}\left(J_{\mathrm{g}} J_{\mathrm{g}}^{\mathrm{T}}\right)\left\{k-\frac{1}{\sigma_{\mathrm{m}}\left(J_{\mathrm{g}} J_{\mathrm{g}}^{\mathrm{T}}\right)}\right\} \leq 0 .
\end{aligned}
$$

Similarly, if $\sigma_{i}<0$, the derivative of the $i$ th part of the candidate Lyapunov function can be evaluated as follows:

$$
\begin{aligned}
\dot{V}_{i} & =\sigma_{i} \dot{\sigma} \leq \sigma_{i}\left\{\left[-k f\left(\boldsymbol{\alpha}, \dot{\theta}_{\mathrm{m}}\right) J_{\mathrm{g}} J_{\mathrm{g}}^{\mathrm{T}} \operatorname{sat}(\boldsymbol{\sigma}, \varepsilon)\right]_{i}-f\left(\boldsymbol{\beta}, \dot{\theta}_{\mathrm{m}}\right)\right\} \\
& \leq \sigma_{i}\left\{f\left(\boldsymbol{\alpha}, \dot{\theta}_{\mathrm{m}}\right)-f\left(\boldsymbol{\beta}, \dot{\theta}_{\mathrm{m}}\right)\right\} \leq 0 .
\end{aligned}
$$

Thus, the following is obtained.

$$
\dot{V}=\sigma^{\mathrm{T}} \dot{\boldsymbol{\sigma}} \leq 0
$$

The reaching mode can be achieved, which means that the sliding vector can reach the state, $\|\sigma\|=\varepsilon$, in a finite time.

\section{A.3. Sliding mode}

The error states governed by $\dot{\boldsymbol{e}}_{i}=-c e_{i}+\sigma_{i}$ after the sliding vector has reached the neighborhood of the sliding manifold at $t=t_{0}$, can be obtained as follows:

$$
\begin{aligned}
e_{i}(t)= & \exp \left(-c\left(t-t_{0}\right)\right) e_{i}\left(t_{0}\right) \\
& +\exp \left(-c\left(t-t_{0}\right)\right) \int_{0}^{t-t_{0}} \sigma_{i} \exp (c \tau) \mathrm{d} \tau .
\end{aligned}
$$

The magnitude of the second term of the right-hand side can be evaluated as follows:

$$
\begin{gathered}
\left\|\exp \left(-c\left(t-t_{0}\right)\right) \int_{0}^{t-t_{0}} \sigma_{i} \exp (c \tau) \mathrm{d} \tau\right\| \\
\leq \varepsilon \frac{1-\exp \left(-c\left(t-t_{0}\right)\right)}{c} \leq \frac{\varepsilon}{c} .
\end{gathered}
$$

Thus, the magnitude of the error states asymptotically decrease and the magnitude of the final error states is less than $\varepsilon / c$. 